\title{
Vasoactive intestinal peptide represses activation of tumor-associated macrophages in gastric cancer via regulation of $\mathrm{TNF} \alpha, \mathrm{IL}-6, \mathrm{IL}-12$ and iNOS
}

\author{
LU CHEN, WEIJIE YUAN, ZHIKANG CHEN, SHAOBIN WU, JIE GE, JINXIANG CHEN and ZIHUA CHEN \\ Department of General Surgery, Xiangya Hospital, Central South University, Changsha 410008, P.R. China
}

Received June 10, 2015; Accepted July 20, 2015

DOI: 10.3892/ijo.2015.3126

\begin{abstract}
Vasoactive intestinal peptide (VIP) has been regarded as deactivator for macrophages. However, the depressive effect of VIP on tumor-associated macrophages (TAM) has not been recognized. In the present study, we investigated the effect of VIP on gastric cancer via TAM by suppressing expression levels of TNF $\alpha$, IL-6, IL-12 and iNOS. Real-time PCR was carried out to examine the expression of CD68 to determine the levels of TAM. The effect of VIP on cell activities was assayed by proliferation assay, colony formation and flow cytometry analysis. The co-culture of TAM and human gastric cancer cell line MKN-45 were performed to understand whether the VIP affects the gastric cancer cells via TAM. Further, the tumor formation in a nude mouse model and VIP injection were performed to illustrate the effect on tumor progression in vivo. CD68 was high expressed in gastric cancer indicating high level of TAM in gastric cancer. Treatment with VIP significantly depressed TAM activation. Moreover, the expression of TNF $\alpha$, IL-6, IL-12 and iNOS in TAM were depressed by VIP treatment, and the VIP treated TAM depressed gastric cancer cells. The experiment in the nude mouse model also suggested that by injection with TAM+VIP, the tumor volume and tumor weight were both decreased significantly. These data suggest that treatment with VIP inhibits gastric cancer.
\end{abstract}

\section{Introduction}

Gastric cancer is one of the most common cancers in the world (1). Up to date, the castration-resistant gastric cancer has only limited curative effect and has shown poor prognosis in clinical practice (2). New treatment strategy of targeting driver pathways provides optional treatment for cancers. For example, induced robust $\mathrm{CD}^{+} \mathrm{T}$ cell response against

Correspondence to: Dr Zihua Chen, Department of General Surgery, Xiangya Hospital, Central South University, 84 Xiangya Road, Changsha 410008, P.R. China

E-mail: chenzihua1@hotmail.com

Key words: vasoactive intestinal peptide, tumor-associated macrophages, gastric cancer, tumor necrosis factor, interleukin, iNOS tumor-associated macrophages (TAM) suggested a novel strategy against breast cancer (3). In tumor progression, TAM is increased and thereby remodels the tumor microenvironment which promoted carcinogenesis (4). By secreting growth and proangiogenic factors, TAM participates in tumor cell proliferation and metastasis via regulating the function of fibroblasts in the tumor stroma (5). Recent studies suggested that anti-TAM effects by small molecule inhibitors could depress tumor suppression, such as cytotoxic (6), biphosphonate compound (7) and zoledronic acid (7). However, these inhibitors showed low infiltrate and limited effects on tumor growth. Thus, the therapeutic targeting of TAM needs to be elucidated and more inhibitors should be developed.

Vasoactive intestinal peptide (VIP) belongs to a superfamily of peptides which also includes pituitary adenylate cyclase-activating polypeptide (PACAP), secretin, and glucagon (8). In recent studies, VIP was shown to regulate the production of TNF $\alpha$, IL-6, IL-12 and iNOS (8-10). Moreover, VIP also inhibits expression levels of cyclooxygenase-2 (COX2) and high mobility group box-1 (HMGB1) in activated macrophages $(11,12)$. In peritonitis, VIP reduced recruitment of neutrophils, macrophages and lymphocytes via controlling the expression of transcription factors including AP-1, CREB and IRF-1 (12-14). Although inhibiting the expression levels of signaling pathways, VIP also induces the expression of toll-like receptors (TLRs) $(15,16)$, indicating the multiple-effects on the immune system. In macrophages, similar to PACAP, VIP was able to bind to specific membrane receptors, including PAC1 and VPAC (17). The receptors of VIP interact with $\mathrm{G}$ proteins, and mediate cAMP-dependent pathway as well as calcium mobilization, protein kinase $\mathrm{C}$, phosphoinositide 3-kinase (PI3-K) and mitogen-activated protein kinase MEK1/2 pathways $(18,19)$. Thus, the expression of VIP in organisms plays a crucial role in multiple biological actions including immunomodulation, muscle relaxation, cell proliferation and differentiation. Several studies have shown that VIP has potential effects on increasing vessel formation in a xenograft model providing insight into VIP treatment in clinical practice $(10,20)$. Similarly, Vacas and colleagues indicated that VIP suppresses clear cell renal cell carcinoma by inducing oxidative stress (9). Therefore, VIP as deactivator of macrophages may also contribute to the suppression of TAM. However, the molecular mechanism underlying this suppression effect of VIP remains poorly understood. 
The effects of VIP on expression of TNF $\alpha$, IL-6, IL-12 and iNOS in macrophages were reported previously (12). TNF $\alpha$, IL-6, IL-12 and iNOS are important regulators and indicators in the process of physiological and pathological immune system (21-23). Herein, we hypothesized that VIP may directly interact with TAM in gastric cancer and modulate the activation of TAM by regulating TNF $\alpha$, IL-6, IL-12 and iNOS. The aim of the present study was to understand the effects of VIP on TAM in gastric cancer and illustrate the mechanism by which VIP represses the activation of TAM. For this purpose, the increasing TAM profile in patients and the depressive effects of VIP on TAM in gastric cancer were studied. Furthermore, by in vivo and in vitro experiments, the TNF $\alpha$, IL-6, IL-12 and iNOS expression levels after VIP treatment was also assayed.

\section{Materials and methods}

Patients. All the samples from gastric cancer were obtained from Xiangya School of Medicine, Central South University (Changsha, China). The experiments in the present study were according to the ethical guidelines of Xiangya School of Medicine Research Ethics Committee. All the patients signed informed consent forms and the study was approved by the Hospital Ethics Committee. In total, 38 patients with gastric cancer were involved in this study. Tissues were collected during the operation. Tissue adjacent to the tumors were determined under a microscope as normal control tissues. The characteristics of the patients were shown in Table I.

Cell culture and treatment. Human gastric cancer cell line MKN-45 was purchased from Shanghai Cell Bank, Chinese Academy of Sciences. TAM was induced from human monocytes THP1 as previously reported (24). Briefly, with 48-h treatment by $320 \mathrm{nmol} / \mathrm{l}$ phorbol myristate acetate (Merck Chemical Division, Rahway, NJ, USA), the suspended cells were transferred into adherent cells. Then the cells were treated with $20 \mathrm{ng} / \mathrm{ml} \mathrm{IL}-4$ and IL-13 for $72 \mathrm{~h}$. The induced TAM was demonstrated using flow cytometry by the biomarkers CD14, CD68, CD206 and CD204.

The MKN-45 cells were cultured in Dulbecco's modified Eagle's medium (DMEM) (Gibco, Gaithersburg, MD, USA) [supplemented with $10 \%$ fetal bovine serum (Gibco)] at $37^{\circ} \mathrm{C}$ in an incubator with atmosphere of $5 \% \mathrm{CO}_{2}$. For TAM, the medium was supplemented with $500 \mathrm{U} / \mathrm{ml}$ IFN- $\gamma$ and $100 \mathrm{ng} / \mathrm{ml}$ LPS (Sigma-Aldrich, St. Louis, MO, USA).

To determine the effect of VIP on TAM, cells were planted at $3 \times 10^{4}$ cells $/ \mathrm{ml}$ in 6 -well plates (Gibco). The 20 wells were randomly divided into five groups $(n=4)$, including $0,0.5,1,2$ and $10 \mu \mathrm{M}$ VIP supplemented groups. After $48-\mathrm{h}$ culture, the TAM responses to the VIP were detected by flow cytometry, proliferation assay and colony formation. The maximal responses of the VIP concentration was confirmed as $1.0 \mu \mathrm{M}$, and used to treat the TAM in following analysis. Subsequently, to understand the effects of TAM and VIP in the treated TAM on the gastric cancer cells, the MKN-45 cells at $3 \times 10^{4}$ cells $/ \mathrm{ml}$ were plated in 6 -well plates (Gibco). The plated cells were divided into five groups $(\mathrm{n}=4)$, including control, TAM+MKN-45, TAM+VIP $(1 \mu \mathrm{M})$, MKN-45+VIP
Table I. The patient characteristics

\begin{tabular}{lc}
\hline Variables & Data \\
\hline Sample size & 38 \\
Age (years) & \\
Median & 53.69 \\
Range & $37-63$ \\
Histology & \\
Distal & 30 \\
Proximal & 8 \\
Size (mm) & \\
$<11$ & 13 \\
$11-20$ & 11 \\
$21-30$ & 7 \\
$30-40$ & 6 \\
$>40$ & 1
\end{tabular}

Histological grade

I

II

III

Stage

I 14

II 12

III $\quad 7$

IV 5

$(1 \mu \mathrm{M})$ and TAM+MKN-45+VIP $(1 \mu \mathrm{M})$. The groups which contained TAM and MKN-45 were co-cultured at concentration of $3 \times 10^{4}$ cells $/ \mathrm{ml}$ for each cell type. For the groups with VIP supplementation, the VIP was added within $48 \mathrm{~h}$ after plating. Subsequently, the cells were analyzed within $96 \mathrm{~h}$. Three independent experiments were performed.

Real-time PCR. Total RNAs from tissues and cells were isolated using RNA TRIzol (Invitrogen, Carlsbad, CA, USA). By agarose gel electrophoresis and BioPhotometer Plus (Eppendorf AG, Hamburg, Germany), the integrity and amount were assayed. Then $2 \mu \mathrm{g}$ of total RNA was reverse transcribed into first cDNA using reverse transcriptase (Invitrogen) according to the manufacturer's protocols. The primers of the present study were designed as shown in Table II. The PCR was performed on ABI 7500 Real-Time PCR system (Applied Biosystems, Austin, TX, USA). The conditions were: $95^{\circ} \mathrm{C}$ for $3 \mathrm{~min}, 40$ cycles at $95^{\circ} \mathrm{C}$ for $12 \mathrm{sec}$ and $55^{\circ} \mathrm{C}$ for $40 \mathrm{sec}$. In this experiment, GAPDH mRNA is the internal control gene for normalization. Tests without DNA template were performed as negative control and melt curves were performed to remove the DNA contamination. The relative expressions of mRNAs were calculated using $2^{-\Delta \Delta C t}$ method.

Western blotting. The protein expression levels were assayed using western blotting. After homogenized in RIPA buffer 
Table II. Primers used for real-time PCR.

\begin{tabular}{lll}
\hline Gene names & \multicolumn{1}{c}{ Forward primers $\left(5^{\prime} \rightarrow 3^{\prime}\right)$} & \multicolumn{1}{c}{ Reverse primers $\left(5^{\prime} \rightarrow 3^{\prime}\right)$} \\
\hline CD68 & CGGAATTCTGCTGGGGCTACTGGCAG & TGATCTAGAGTCCCCTGGGCTTTTGGCAG \\
TNF- $\alpha$ & GGAGAAGGGTGACCGACTCA & CTGCCCAGACTCGGCAA \\
IL-6 & AGCACATTAAGTACATCCTCGGC & CCAGATTGGAAGCATCCGTC \\
IL-12 & TGGAGTGCCAGGAGGACAGT & TCTTGGGTGGGTCAGGTTTG \\
iNOS & GGATGACTTTCGAGGACATGC & GGGCCCTCTGGTCATACTTTT \\
GAPDH & TGCACCACCAACTGCTTAGC & GGCATGGACTGTGGTCATGAG
\end{tabular}

(50 mM Tris pH 8.0, $150 \mathrm{mM} \mathrm{NaCl}, 1 \%$ NP-40, 0.5\% DOC, $0.1 \%$ SDS, $1 \mathrm{mM}$ DTT, protease and phosphatase inhibitors), the protein were isolated and then the quantity was determined by BCA protein assay kit (Beyotime, Wuhan, China). For each sample, $20 \mu \mathrm{g}$ total protein was separated by $10 \%$ dodecyl sulfate polyacrylamide gel. The protein on gel was then transferred into polyvinylidene fluoride membranes (Millipore, Billerica, MA, USA). After blocking with $4 \%$ skim milk for $1 \mathrm{~h}$ at room temperature, primary polyclonal antibodies of CD68 (IS613, Dako, Denmark, produced in rabbit, 1:1,000), TNF $\alpha$ (T8300, Sigma-Aldrich, produced in rabbit, 1:2,000), IL-6 (MFCD00162579, Sigma-Aldrich, produced in rabbit, 1:1,000), IL-12 (I4153, Sigma-Aldrich, produced in goat, 1:1,000), iNOS (SAB4502011, Sigma-Aldrich, produced in rabbit, 1:1,000) and GAPDH (SAB2100894, Sigma-Aldrich, produced in rabbit, 1:500) were incubated with membranes at $4{ }^{\circ} \mathrm{C}$ overnight. All the antibodies were purchased from Sigma-Aldrich. After 3 times washing in TBST buffer (pH 7.6, 20 mM Tris-HCl, $137 \mathrm{mM} \mathrm{NaCl,} \mathrm{0.01 \%} \mathrm{Tween-20),}$ the second antibodies (HRP-conjugated anti-rabbit IgG and HRP-conjugated anti-goat IgG, Sigma-Aldrich, 1:2,000) and enhanced chemiluminescence (ECL, Millipore) were used to visualized the protein signals.

Immunohistochemical staining (IHC). Tissues were cut into $6-\mu \mathrm{m}$ thick sections in paraffin wax. After de-waxing, the sections were blocked with $4 \%$ skim milk and incubated with primary antibodies (CD68, 1:200; TNF $\alpha$, 1:200; IL-6, 1:500; IL-12, 1:200; iNOS, 1:200) at $4^{\circ} \mathrm{C}$ overnight. Subsequently, the proteins were visualized after the second antibody incubation at room temperature for $1 \mathrm{~h}$ and visualized using streptavidinbiotinylated horseradish peroxidase complex kit (Beyotime). The TAM and MKN-45 cells were labeled by antibodies of FITC-CD68 (1:200) and cy5-MSI1 (1:200) (Bioss Co., China). Each staining was repeated 3 times.

Colony formation assay. After treatment, 2,000 cells from each group were plated into 6-well plates and incubated for 7 days at $37^{\circ} \mathrm{C}$ in an incubator with atmosphere of $5 \% \mathrm{CO}_{2}$. After washing with phosphate buffer solution (PBS) three times, the cells were fixed with methanol for $15 \mathrm{~min}$ and stained using $0.2 \%$ crystal violet for $15 \mathrm{~min}$. The colonies were counted and mean values were calculated from three independent experiments.

Flow cytometry. To analyze the apoptosis of the cells, we used flow cytometry (Coulter, Luton, UK) and Annexin V-FITC and propidium iodide (PI) (BioVision, Milpitas, CA, USA) staining according to the manufacturer's instructions. At least 30,000 cells for each sample were treated. The detection of biomarkers of TAM was performed using antibodies of CD14, CD68, CD206 and CD204. The cells were fixed using Cytofix/Cytoperm ${ }^{\mathrm{TM}}$ Fixation/Permeabilization solution (BD Biosciences, Franklin Lakes, NJ, USA). The FITC-CD68, FITC-CD14, PE-CD206, and PE-CD204 antibodies (SigmaAldrich) were incubated with the cells and labeled with PE-conjugated goat anti-mouse secondary antibody. Triplicate biological repeats were measured for this experiment.

Tumor formation in a nude mouse model. We used 5-week-old nude mice to generate the tumor model. The animal experiments were approved of the ethics committees of Xiangya School of Medicine, Central South University (Changsha, China). The tested mice were randomly divided into three groups ( $\mathrm{n}=10$ for each group), including control, VIP and VIP+TAM group. The mice were first injected with $3 \times 10^{4}$ MKN-45 cells in $1 \mathrm{ml}$ DMEM. The injections were performed every 2 days for 20 days until the tumor size was $100 \mathrm{~mm}^{3}$. The tumor size was calculated as $\mathrm{V}=\mathrm{LxWxDx3.14/6}$. Subsequently, TAM and TAM+VIP $(1 \mu \mathrm{M})$ were injected into the tumor tissues. Also, a blank group was performed by injection with saline. The tumor volumes were measured every 2 days until 20 days. After 20 days, the tumor tissues were excised and assayed using real-time PCR, western blotting and IHC.

Statistical analysis. The data are indicated as mean \pm standard deviation (SD). The difference among the groups were confirmed using one-way-ANOVA analysis. The significant difference was determined at $\mathrm{P}<0.05$. All the statistical analysis were performed using SPSS 17.0 (SPSS Inc., Chicago, IL, USA).

\section{Results}

Increasing of TAM in gastric cancer. To assess the TAM level in gastric cancer, the indicator of TAM, CD68 was detected using real-time PCR. Compared to the adjacent normal tissues, the expression of CD68 was much higher in cancer samples $(\mathrm{P}<0.001$, Fig. 1A). High level of CD68 was observed in the high histological grade (Fig. 1B) and advanced tumor stage (Fig. 1C). The results indicated high levels of TAM in gastric cancer.

VIP depresses TAM activation. The TAM were induced by THP1 human monocytes. The flow cytometry analysis of biomarkers including CD14 (marker for monocyte differentia- 

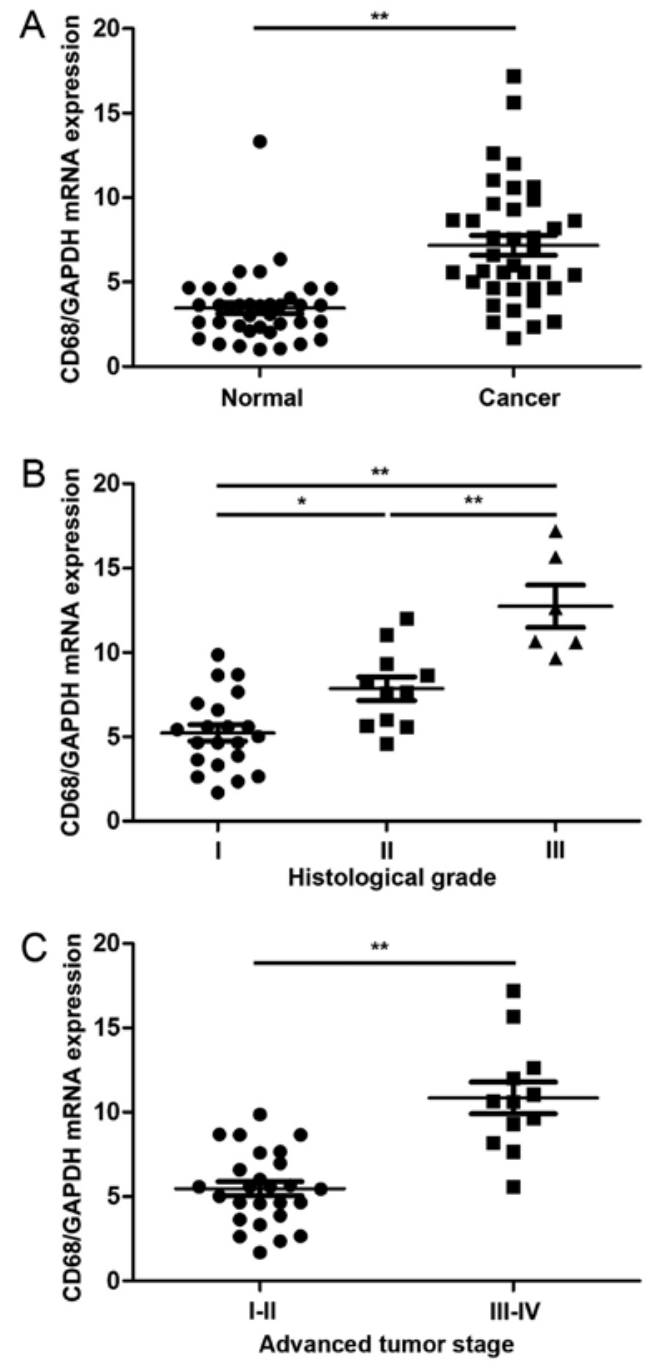

Figure 1. Expression of CD68 is upregulated in gastric cancer. (A) Compared to the normal tissues, the expression of CD68 was much higher in the gastric cancer tissues. (B) Higher expression of CD68 was observed based on the histological grade. (C) The expression of CD68 was much higher in III-IV advanced tumor stages compared to I-II advanced tumor stages. " $\mathrm{p}<0.05 ;{ }^{* *} \mathrm{p}<0.001$.

tion), CD68 (marker for macrophages differentiation), CD206, and CD204 (both markers for M2 macrophages) proved that the TAM were successfully induced (Fig. 2).

After VIP treatment, the CD68 mRNA levels in TAM were significantly depressed $(\mathrm{P}<0.05)$. The 0.5 and $1.0 \mu \mathrm{M}$ VIP treatment showed the most significant effects on expres- sion of CD68 mRNA indicating the inhibition of TAM by VIP (Fig. 3A). The protein expression levels decreased significantly and was similar to the expression of mRNA. The $1.0 \mu \mathrm{M}$ VIP treatment showed the most efficient depressive effects (Fig. 3B). The colony formation assay suggested that treatment with 1.0 $\mu \mathrm{M}$ VIP inhibited growth of TAM (Fig. 3C). By flow cytometry, we also showed that VIP treatment stimulated the apoptosis of TAM and 1.0 $\mu \mathrm{M}$ VIP induced apoptosis with the optimal dosage (Fig. 3D).

VIP inhibits TNF $\alpha, I L-6, I L-12$ and iNOS in TAM. VIP has been shown to depress expression of TNF $\alpha$, IL-6, IL-12 and iNOS in macrophages. In the present study, the effect of VIP on expression of TNFa, IL-6, IL-12 and iNOS in TAM was determined. The result showed that VIP depressed the expression of TNF $\alpha$, IL- 6 and IL-12 in all the treatment groups, including $0.5,1.0,2.0$ and $10.0 \mu \mathrm{M}$ VIP treatment (Fig. 4A-C). For iNOS, except the $0.5 \mu \mathrm{M}$ VIP treatment, the other concentrations of VIP treatment, including 1.0, 2.0 and $10.0 \mu \mathrm{M}$ VIP, inhibited the expression in TAM significantly (Fig. 4D). Western blotting showed similar changes (Fig. 4E). The protein expression of TNFa, IL-6, IL-12 and iNOS was dcreased after VIP treatment. The $1.0 \mu \mathrm{M}$ VIP treatment group showed inhibition of these genes among all the groups, thus, the following experiment was performed using $1.0 \mu \mathrm{M}$ VIP in treatment of TAM.

The VIP-treated TAM depressed gastric cancer cells. To examine the possible effects of VIP on gastric cancer cells via TAM, TAM were co-cultured with human gastric cancer cell line MKN-45 and treated with VIP. The co-culture of TAM and MKN-45 is shown in Fig. 5A. The CD68 and MSI1 as specific biomarkers for TAM and MKN-45 were used to identify the cells. The result showed co-existence of TAM and MKN-45 (Fig. 5A). Colony formation assay showed that the VIP-treated TAM remarkably reduced colony formation of gastric cancer cells (Fig. 5B). Moreover, without TAM, the MKN-45+VIP group had no significant decrease compared to control, which indicated the depressive effects of VIP on gastric cancer cells is indirect and meditated by TAM (Fig. 5B). The proliferation assay showed that the lowest cell viability of MKN-45 cells was observed in TAM+VIP and TAM+MKN-45+VIP group (Fig. 5C). The TAM+MKN-45 group had the highest cell viability, while, other groups showed medial cell viability. Apoptosis demonstrated by flow cytometry showed similar results. TAM+MKN-45+VIP group
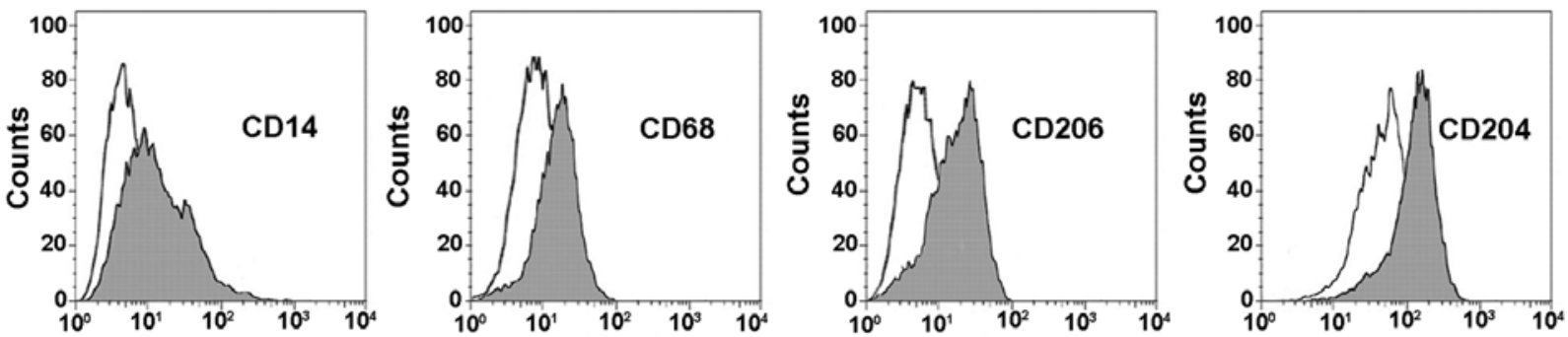

Figure 2. Confirmation of induced TAM by CD14 (marker for monocyte differentiation), CD68 (marker for macrophage differentiation), CD206, and CD204 (both markers for M2 macrophages). 


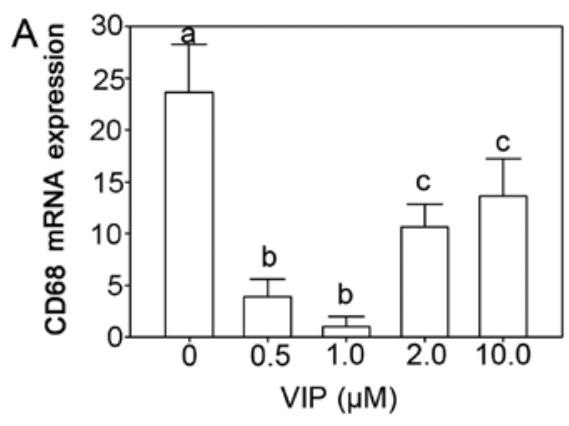

B

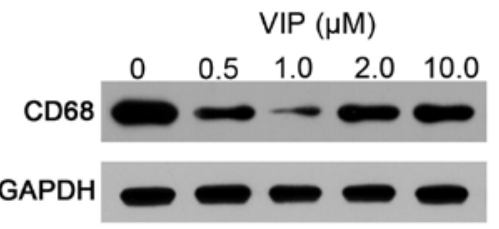

C
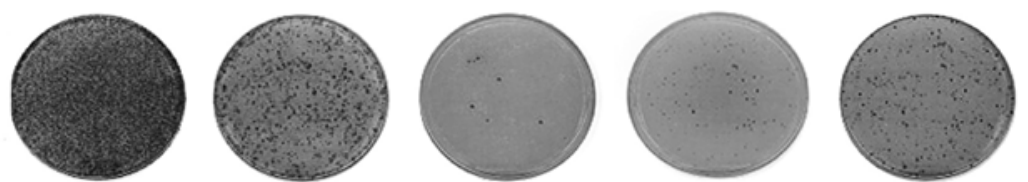

0 VIP $(\mu \mathrm{M})$

0.5 VIP $(\mu \mathrm{M}) \quad 1.0$ VIP $(\mu \mathrm{M})$

2.0 VIP $(\mu \mathrm{M}) \quad 10.0$ VIP $(\mu \mathrm{M})$
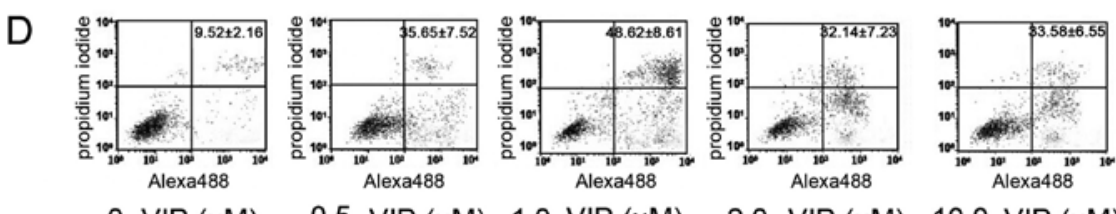

0 VIP $(\mu \mathrm{M})$

$0.5 \mathrm{VIP}(\mu \mathrm{M}) \quad 1.0 \mathrm{VIP}(\mu \mathrm{M})$

2.0 VIP $(\mu \mathrm{M})$

10.0 VIP $(\mu \mathrm{M})$

Figure 3. VIP depresses activation of TAM. (A) Real-time PCR shows inhibition of CD68 mRNA levels of TAM by VIP ( $\mathrm{n}=4$ for each group, data are expressed as the means $\pm \mathrm{SD}$; different lower case characters represent significant differences, $\mathrm{P}<0.05$ ). (B) Western blotting indicates the depressive effects of VIP on protein levels of CD68. (C) Colony formation test of TAM after VIP treatment. (D) Flow cytometry assay indicates that VIP treatment induces apoptosis of TAM. The same letter indicates no significant difference while different letter superscripts represent significant difference.
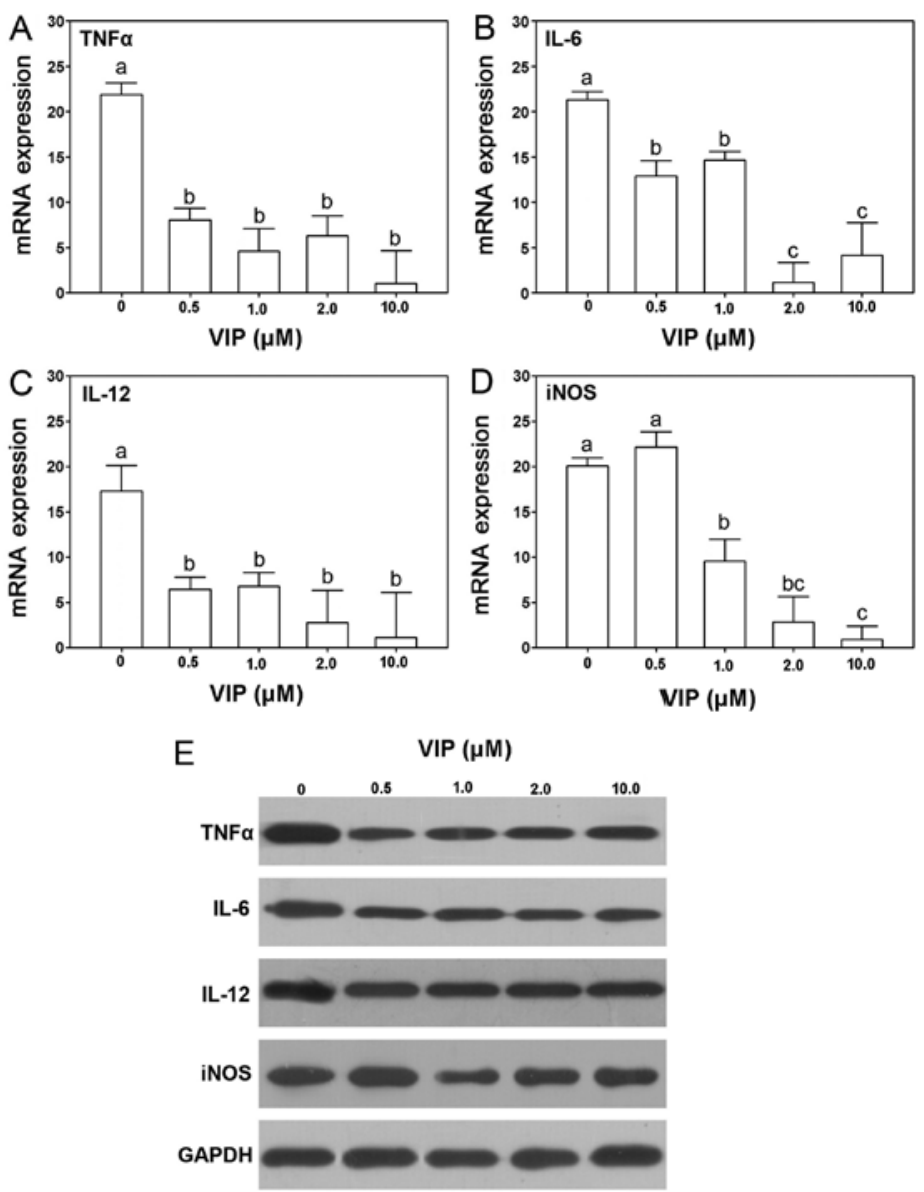

Figure 4. VIP inhibits expression of TNFa, IL-6, IL-12 and iNOS in TAM. Real-time PCR indicates that mRNA expression of TNF $\alpha$ (A), IL-6 (B), IL-12 (C) and iNOS (D) of TAM ( $n=4$ for each group, data are expressed as the means $\pm S D$; different lower case characters represent significant differences, $P<0.05$ ). (E) Western blotting also shows depressive effects of VIP on protein expression of TNFa, IL-6, IL-12 and iNOS in TAM. 
A
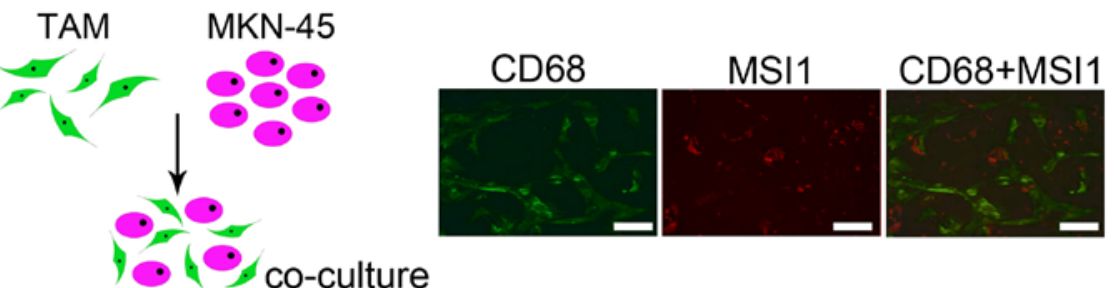

B
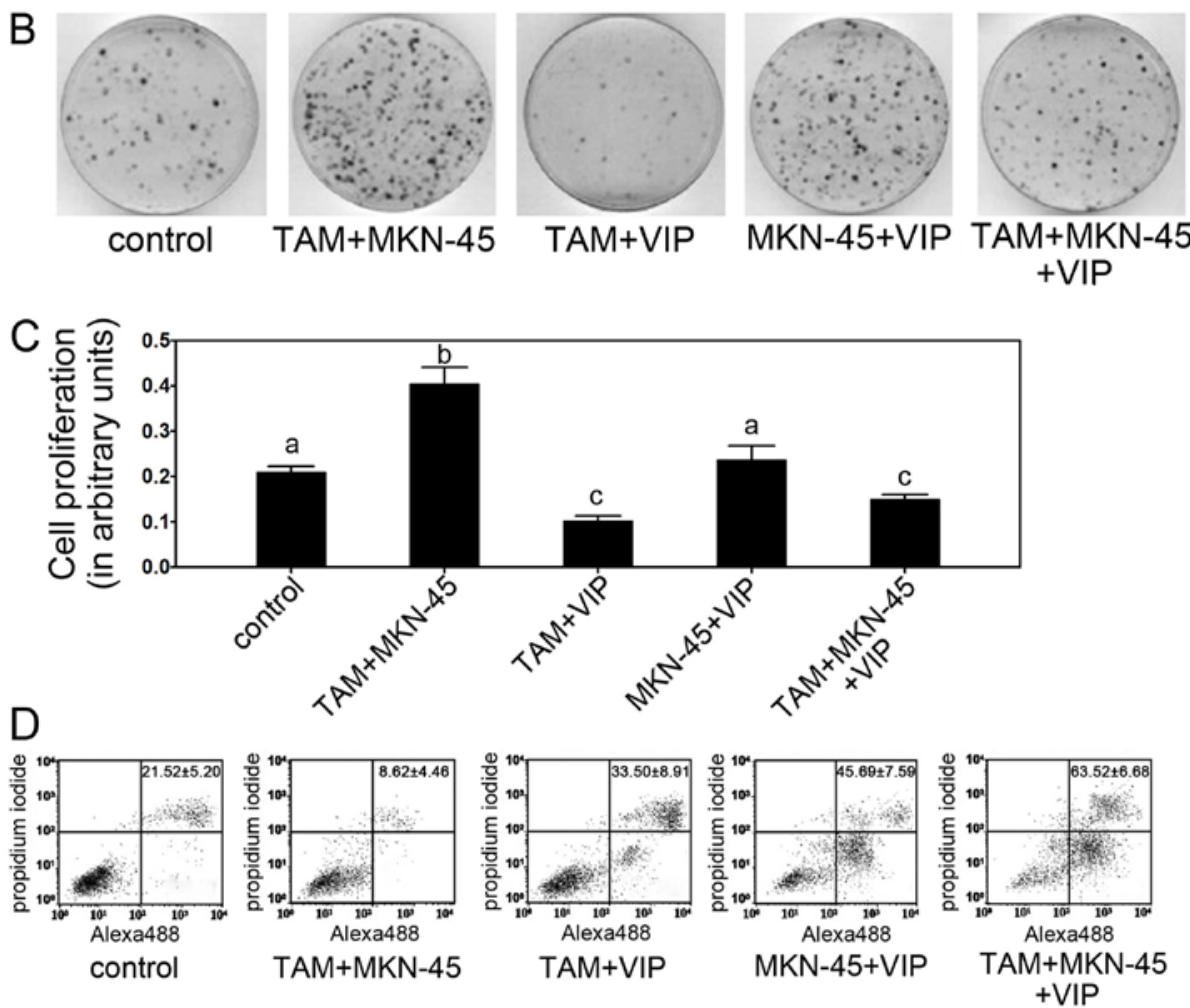

Figure 5. VIP inhibits activation of gastric cancer cells via depressing TAM. (A) Co-culture of TAM and MKN-45. CD68 and MSI1 are labeled TAM and MKN-45, respectively. (B) Colony formation test of gastric cancer cells (MKN-45) and TAM after VIP treatment. (C) Cell proliferation of gastric cancer cells (MKN-45) and TAM after VIP treatment ( $\mathrm{n}=4$ for each group, data are expressed as the means \pm SD; different lower case characters represent significant differences, $\mathrm{P}<0.05)$. (D) Flow cytometry assay indicates apoptosis of gastric cancer cells (MKN-45) and TAM after VIP treatment. The same letter indicates no significant difference while different letter superscripts represent significant difference.

showed the highest apoptosis rate while TAM+MKN-45 group had the lowest apoptosis rate (Fig. 5D).

As the VIP depressed activation of gastric cancer cells via TAM, we next determine if VIP affects gene expression levels in cultured gastric cancer cells directly or indirectly. VIP downregulated TNF $\alpha$, IL-6, IL-12 and iNOS were found in TAM+VIP and TAM+MKN-45+VIP groups while MKN-45+VIP group showed no significant difference of expression compared to control, which suggested that the depressive effects of TNF $\alpha$, IL-6, IL-12 and iNOS was mediated by TAM (Fig. 6).

VIP depresses TAM and tumor formation in the nude mouse model. To illustrate the effect of VIP on gastric cancer in vivo, the tumor formation in the nude mouse model was constructed by $3 \times 10^{4} \mathrm{MKN}-45$ cell injections. After tumor formation, the TAM and TAM+VIP were injected into the tumors. The tumor volume and tumor weight of TAM+VIP group were significantly lower compared with the TAM group $(\mathrm{P}<0.05$ after 20 days) (Fig. 7A and B). The CD68 was increased accordingly in TAM groups while depressed CD68 was found in TAM+VIP group indicating the depressive effect of TAM in vivo (Fig. 7C-E).

Consistent with the result in vitro, the expression levels of TNF $\alpha$, IL-6, IL-12 and iNOS in xenograft tumor tissues were downregulated by VIP in TAM+VIP group compared with the TAM group (Fig. 8). Thus, the results of the xenograft model suggested that VIP inhibits the tumor progression of gastric cancer mediated by TAM in vivo via downregulating expression of TNF $\alpha$, IL-6, IL-12 and iNOS.

\section{Discussion}

The present study showed the primary findings on the VIP depressive effect on TAM. Moreover, the treatment with VIP inhibits the expression of TNF $\alpha$, IL-6, IL-12 and iNOS in TAM, which results in deactivation of TAM. For cancer cells, TAM acts as mediators for interacting growth factors, cytokines and chemokines and change the tumor microenvironment to stimulate tumor progression (25-27). We demonstrated that the VIP inhibited gastric cancer via TAM both in cultured cells and in the nude mouse model. 

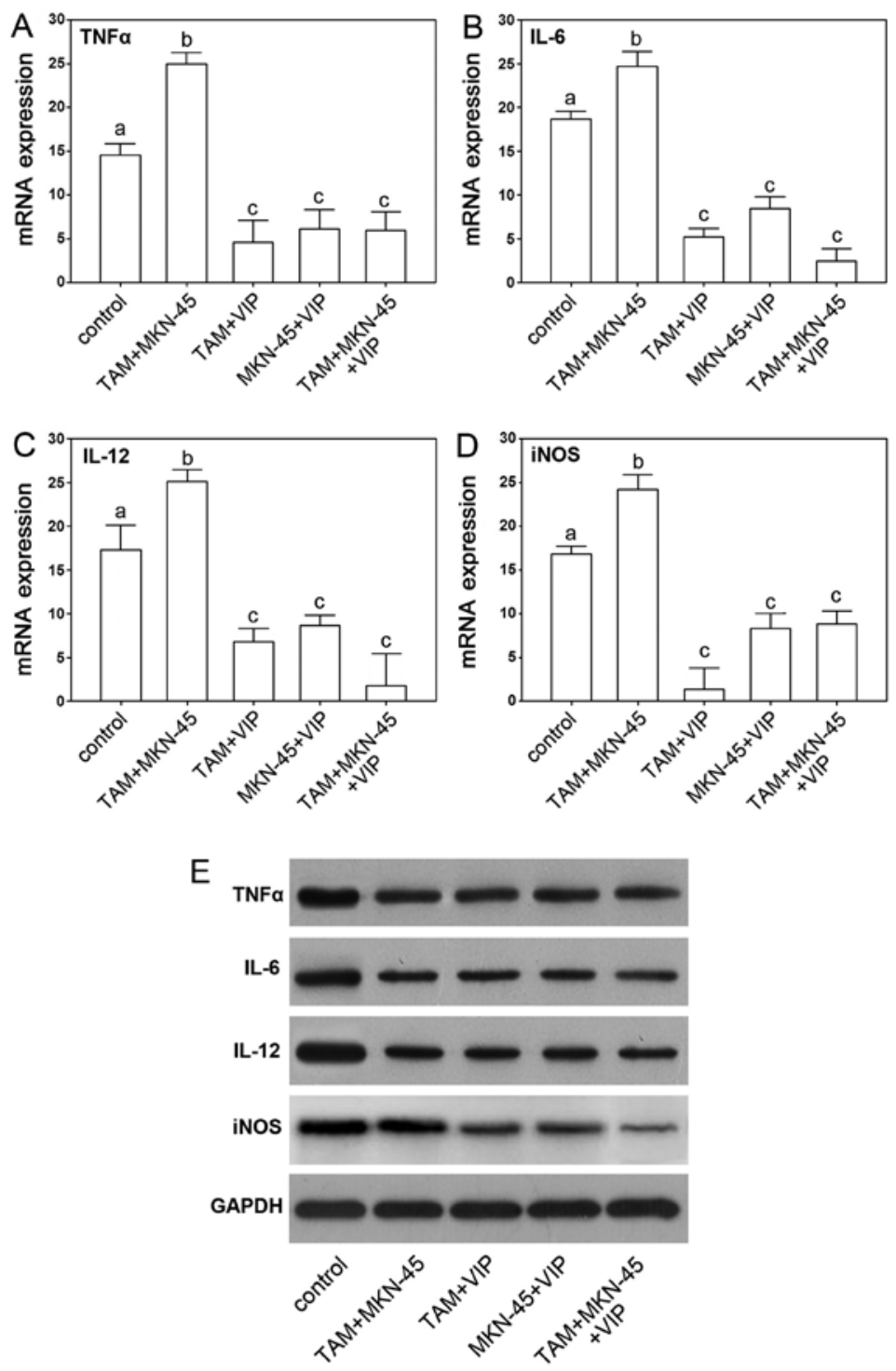

Figure 6. VIP depression of TNF $\alpha$, IL-6, IL-12 and iNOS after VIP treatment in TAM co-cultured with gastric cancer cells. Real-time PCR indicates that mRNA expression of TNF $\alpha$ (A), IL-6 (B), IL-12 (C) and iNOS (D) of TAM co-cultured with gastric cancer cells (n=4 for each group, data are expressed as the means $\pm \mathrm{SD}$; different lower case characters represent significant differences, $\mathrm{P}<0.05$ ). (E) Western blotting also shows depressive effects of VIP on protein expression of TNF $\alpha$, IL-6, IL-12 and iNOS in TAM co-cultured with gastric cancer cells.

Macrophages originating from blood monocytes are divided into M1 (classically activated) and M2 types (alternatively activated) (28). TAM has been regarded as M2 phenotype and play mostly pro-tumoral functions such as promoting tumor cell survival, proliferation and invasion (29). High levels of TAM are correlated with poor prognosis (30). Simultaneously, CD68 as indicator of TAM has been used as molecular signature to determine the prognosis of cancer $(31,32)$. With no surprise, as we found in the present study, CD68 was highly expressed in gastric cancer tissues compared to normal tissues showing similar results with previous reports with higher level of TAM in tumor than normal tissues. Accordingly, depression of TAM may be a potential therapy for gastric cancer treatment. Further, we used VIP, a pleiotropic peptides to intervene in TAM to demonstrate the possibility of VIP as new therapeutic strategy.

Tumorigenesis as a complex process, results from molecular and cellular variation with a variety of compounds including oncoproteins and tumor proteins. During this process, TAM are the promotional factor for tumorigenesis (33). VIP is a neuropeptide that exerts multiple actions in different types of cells (8). Several studies showed that the de-activated function of VIP in macrophages $(11,12,34)$. As we found in the present study, VIP depressed TAM as well. It is known that VIP affects the expression of both pro- and anti-inflammatory factors after LPS and IFN $\gamma$ induced macrophages $(35,36)$. In the present study, the treatment of VIP depressed activities of TAM by suppressing expression of CD68 and colony formation as well as inducing apoptosis. Based on these findings, it seems likely that the depressing effect of VIP on the proliferation of macrophages also exists in TAM. However, there is still a lack of straight forward answer as to how VIP suppressed the TAM and whether VIP could inhibit tumorigenesis by depressing TAM in vivo.

The molecular mechanism by which VIP exerts its deactivated effects in macrophage is well studied. VIP has been 

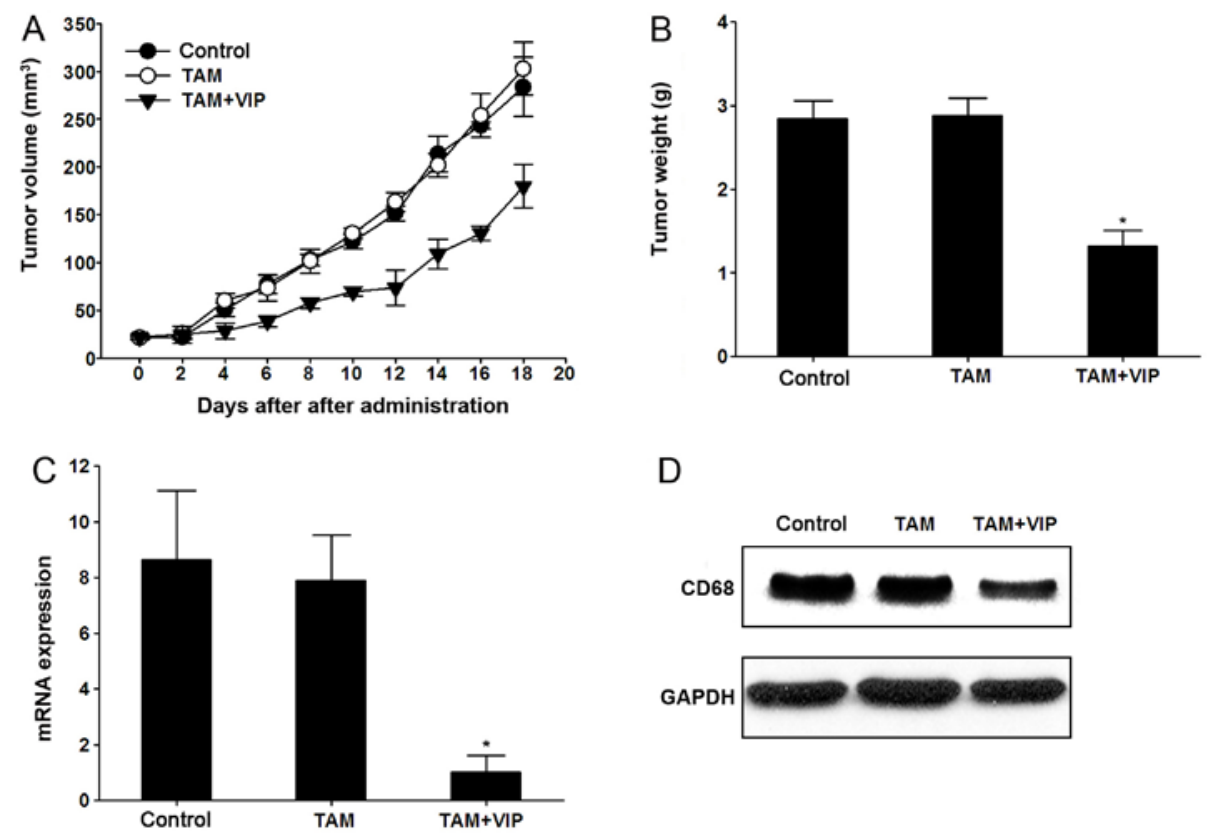

D
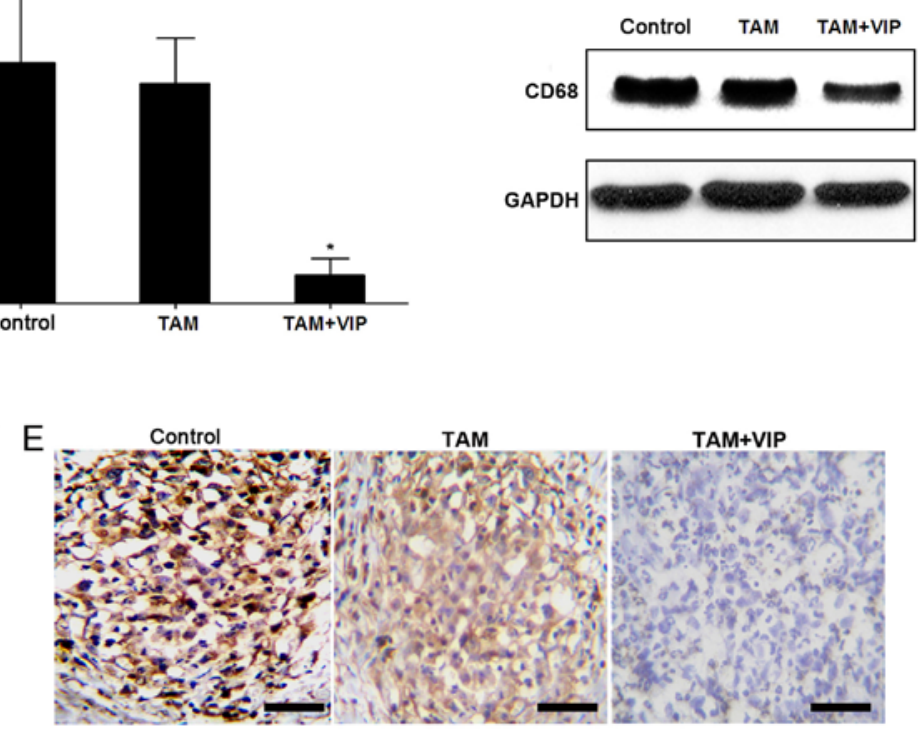

Figure 7. VIP treatment of TAM depresses tumor formation. (A) Tumor volume changes after injection with TAM and VIP treated TAM (n=10 mice in each group). (B) Tumor weight after injection with TAM and VIP treated TAM ( $\mathrm{n}=10$ mice in each group, data are expressed as the means \pm SD; asterisks showed significant difference between the groups, $\mathrm{P}<0.05$ ). (C) Expression of CD68 mRNA in xenograft tumors after injection with TAM and VIP treated TAM ( $\mathrm{n}=5$ mice in each group, data are expressed as the means $\pm \mathrm{SD}$; asterisks showed significant difference between the groups, $\mathrm{P}<0.05)$. (D) Expression of $\mathrm{CD} 68$ protein in xenograft tumors after injection with TAM and VIP treated TAM assayed by western blotting (data are expressed as the means \pm SD; asterisks show significant difference between the groups, P<0.05). (E) Distribution and expression of CD68 detected by IHC. Bar, $50 \mu \mathrm{m}$.
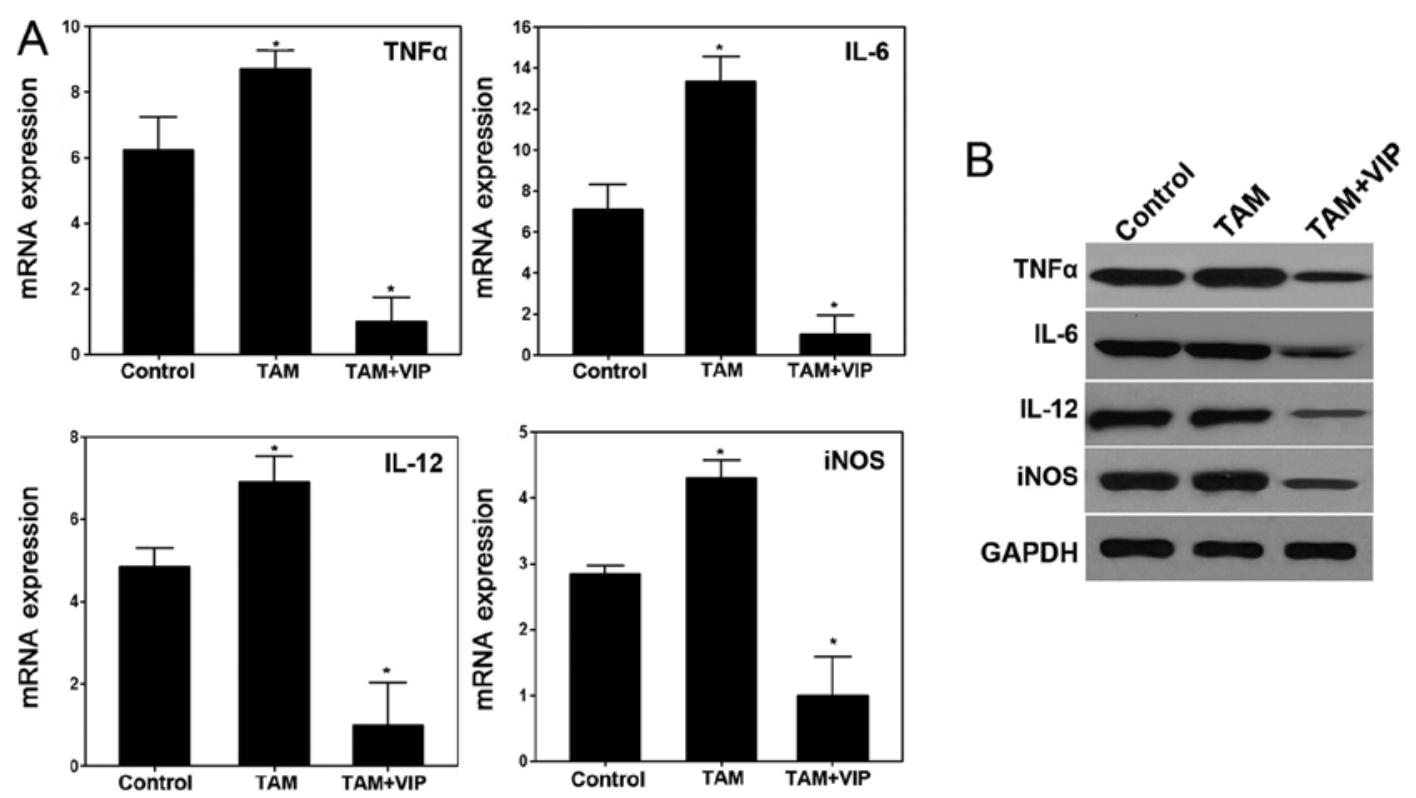

Figure 8. Expression of TNF $\alpha$, IL-6, IL-12 and iNOS in xenograft tumors after injection with TAM and VIP treated TAM. (A) Real-time PCR result indicates depressive effect of VIP treatment on mRNA expression of TNF $\alpha$, IL-6, IL-12 and iNOS in xenograft tumors after TAM injection ( $\mathrm{n}=5$ mice in each group, data are expressed as the means \pm SD; asterisks show significant difference between the groups, $\mathrm{P}<0.05$ ). (B) Western blotting shows depressive effect of VIP treatment on protein expression of TNFa, IL-6, IL-12 and iNOS in xenograft tumors after TAM injection. 
shown to regulate the expression and/or transactivating of transcription factors such as AP-1, NFkB, CREB and IRF-1 $(13,14,34,36)$ and mediate the expression of chemokines, tumor necrosis factors, $\mathrm{COX} 2$, interleukin and toll-like receptors $(8,15)$. TNF $\alpha$ showed tumor-promoting roles in previous studies and is regarded as a target in malignant cancer $(21,37)$. Inhibition of TNF $\alpha$ reduces metastatic activity in tumors (37). The present study showed that VIP inhibited production of TNF $\alpha$ and the incubation of VIP suppressed the effects of TAM. These data are consistent with previous studies showing that VIP reduced the growth of macrophage via regulating the growth factor, and in macrophages, IL-6, IL-12 and iNOS could be inhibited by VIP which showed deactivation effects of VIP on macrophages $(8,11,12)$. IL-6 and IL-12 are synthesized by macrophages and participate in inducing antibody secretion, acute phase reaction, hematopoiesis and regulating production of IFN- $\gamma$ and TNF $\alpha$ (8). iNOS, also participates in the immune response by binding to calmodulin and produces $\mathrm{NO}$ as an immune defense mechanism which also indicates the activities of macrophages (23). The inhibited expression levels of IL-6, IL-12 and iNOS by VIP showed decreased activity of TAM. These results indicate that treatment with VIP inhibits TNF $\alpha$ and IL-6, IL-12 and iNOS of TAM which then results in depressed cell proliferation.

Our data showed that VIP inhibits progression of TAM, and the role of TAM as cancer promoter has been demonstrated previously. This evidence suggests TAM as potential therapeutic target in human cancer and VIP could be an efficient inhibitor for TAM. In the present study, VIP is found to inhibit gastric cancer cells as well as tumor formation in the nude mouse model. We found the depressive effects of VIP are indirect via first suppressing TAM. The decreased expression of TNF $\alpha$ and IL-6, IL-12 and iNOS after VIP treated required co-culture of TAM and $\mathrm{MKN}-45$. In a previous study, VIP suppressed metastatic human clear cell renal cell carcinoma by inducing oxidative stress (9). Vacas et al demonstrated that VIP inhibited invasion and metastasis of human clear cell renal cell carcinoma via decreasing $\beta$-catenin (9). On the contrary, high expression of VIP in pancreas could induce VIPoma which is a very rare type of cancer that usually derived from pancreatic cells (38). Thus, the roles of VIP in cancer progression seem contradictory in different types of cancer which needs to be elucidated in further study. The results of our present study showed that the VIP treatment with a proper dosage could inhibit progression of gastric cancer by deactivating TAM which is similar to the macrophages by decreasing expression levels of TNF $\alpha$ and IL-6, IL-12 and iNOS.

In conclusion, we demonstrated that VIP inhibits progression of gastric cancer mediated by TAM in the present study. The antitumor action of VIP appears to be initiated by interaction with TAM via depression of the expression levels of TNF $\alpha$ and IL-6, IL-12 and iNOS. The presented data provide new insight into the therapeutic application of VIP to inhibit gastric cancer both in vivo and in vitro via TAM.

\section{References}

1. Roder DM: The epidemiology of gastric cancer. Gastric Cancer 5 (Suppl 1): S5-S11, 2002.
2. Cervantes A, Roda D, Tarazona N, Roselló S and PérezFidalgo JA: Current questions for the treatment of advanced gastric cancer. Cancer Treat Rev 39: 60-67, 2013.

3. Mukhtar RA, Nseyo O, Campbell MJ and Esserman LJ: Tumorassociated macrophages in breast cancer as potential biomarkers for new treatments and diagnostics. Expert Rev Mol Diagn 11: 91-100, 2011.

4. Noy R and Pollard JW: Tumor-associated macrophages: From mechanisms to therapy. Immunity 41: 49-61, 2014.

5. De Palma M and Lewis CE: Macrophage regulation of tumor responses to anticancer therapies. Cancer Cell 23: 277-286, 2013.

6. Bingle L, Brown NJ and Lewis CE: The role of tumour-associated macrophages in tumour progression: Implications for new anticancer therapies. J Pathol 196: 254-265, 2002.

7. Giraudo E, Inoue M and Hanahan D: An amino-bisphosphonate targets MMP-9-expressing macrophages and angiogenesis to impair cervical carcinogenesis. J Clin Invest 114: 623-633, 2004.

8. Delgado $\mathrm{M}$ and Ganea D: Vasoactive intestinal peptide: A neuropeptide with pleiotropic immune functions. Amino Acids 45: 25-39, 2013.

9. Vacas E, Bajo AM, Schally AV, Sánchez-Chapado M, Prieto JC and Carmena MJ: Vasoactive intestinal peptide induces oxidative stress and suppresses metastatic potential in human clear cell renal cell carcinoma. Mol Cell Endocrinol 365: 212-222, 2013.

10. Yang J, Shi QD, Song TB, Feng GF, Zang WJ, Zong CH and Chang L: Vasoactive intestinal peptide increases VEGF expression to promote proliferation of brain vascular endothelial cells via the cAMP/PKA pathway after ischemic insult in vitro. Peptides 42: 105-111, 2013.

11. Delgado M and Ganea D: Vasoactive intestinal peptide prevents activated microglia-induced neurodegeneration under inflammatory conditions: Potential therapeutic role in brain trauma. FASEB J 17: 1922-1924, 2003.

12. Delgado M, Robledo G, Rueda B, Varela N, O'Valle F, HernandezCortes P, Caro M, Orozco G, Gonzalez-Rey E and Martin J: Genetic association of vasoactive intestinal peptide receptor with rheumatoid arthritis: Altered expression and signal in immune cells. Arthritis Rheum 58: 1010-1019, 2008.

13. Delgado $M$ and Ganea D: Cutting edge: Is vasoactive intestinal peptide a type 2 cytokine? J Immunol 166: 2907-2912, 2001.

14. Delgado $M$ and Ganea D: Inhibition of endotoxin-induced macrophage chemokine production by VIP and PACAP in vitro and in vivo. Arch Physiol Biochem 109: 377-382, 2001.

15. Gomariz RP, Arranz A, Abad C, Torroba M, Martinez C, Rosignoli F, Garcia-Gómez M, Leceta J and Juarranz Y: Timecourse expression of Toll-like receptors 2 and 4 in inflammatory bowel disease and homeostatic effect of VIP. J Leukoc Biol 78: 491-502, 2005.

16. Arranz A, Juarranz Y, Leceta J, Gomariz RP and Martínez C: VIP balances innate and adaptive immune responses induced by specific stimulation of TLR2 and TLR4. Peptides 29: 948-956, 2008.

17. Laburthe M and Couvineau A: Molecular pharmacology and structure of VPAC Receptors for VIP and PACAP. Regul Pept 108: 165-173, 2002.

18. Brenneman DE: Neuroprotection: A comparative view of vasoactive intestinal peptide and pituitary adenylate cyclase-activating polypeptide. Peptides 28: 1720-1726, 2007.

19. Voice JK, Dorsam G, Chan RC, Grinninger C, Kong Y and Goetzl EJ: Immunoeffector and immunoregulatory activities of vasoactive intestinal peptide. Regul Pept 109: 199-208, 2002.

20. Collado B, Carmena MJ, Clemente C, Prieto JC and Bajo AM: Vasoactive intestinal peptide enhances growth and angiogenesis of human experimental prostate cancer in a xenograft model. Peptides 28: 1896-1901, 2007.

21. Balkwill F: TNF- $\alpha$ in promotion and progression of cancer. Cancer Metastasis Rev 25: 409-416, 2006.

22. Wong CK, Ho CY, Ko FW, Chan CH, Ho AS, Hui DS and Lam CW: Proinflammatory cytokines (IL-17, IL-6, IL-18 and IL-12) and Th cytokines (IFN- $\gamma$, IL-4, IL-10 and IL-13) in patients with allergic asthma. Clin Exp Immunol 125: 177-183, 2001.

23. Aktan F: iNOS-mediated nitric oxide production and its regulation. Life Sci 75: 639-653, 2004.

24. Tjiu JW, Chen JS, Shun CT, Lin SJ, Liao YH, Chu CY, Tsai TF, Chiu HC, Dai YS, Inoue H, et al: Tumor-associated macrophageinduced invasion and angiogenesis of human basal cell carcinoma cells by cyclooxygenase-2 induction. J Invest Dermatol 129: 1016-1025, 2009. 
25. Liu J, Zhang N, Li Q, Zhang W, Ke F, Leng Q, Wang H, Chen J and Wang H: Tumor-associated macrophages recruit $\mathrm{CCR}^{+}$ regulatory $\mathrm{T}$ cells and promote the development of colorectal cancer via enhancing CCL20 production in mice. PLoS One 6: e19495, 2011.

26. Caillou B, Talbot M, Weyemi U, Pioche-Durieu C, Al Ghuzlan A, Bidart JM, Chouaib S, Schlumberger M and Dupuy C: Tumorassociated macrophages (TAMs) form an interconnected cellular supportive network in anaplastic thyroid carcinoma. PLoS One 6: e22567, 2011.

27. Chen JJ, Lin YC, Yao PL, Yuan A, Chen HY, Shun CT, Tsai MF, Chen CH and Yang PC: Tumor-associated macrophages: The double-edged sword in cancer progression. J Clin Oncol 23: 953-964, 2005.

28. Martinez FO, Sica A, Mantovani A and Locati M: Macrophage activation and polarization. Front Biosci 13: 453-461, 2008.

29. Mantovani A, Sozzani S, Locati M, Allavena P and Sica A: Macrophage polarization: Tumor-associated macrophages as a paradigm for polarized M2 mononuclear phagocytes. Trends Immunol 23: 549-555, 2002.

30. Pollard JW: Tumour-educated macrophages promote tumour progression and metastasis. Nat Rev Cancer 4: 71-78, 2004.

31. Khorana AA, Ryan CK, Cox C, Eberly S and Sahasrabudhe DM: Vascular endothelial growth factor, CD68, and epidermal growth factor receptor expression and survival in patients with Stage II and Stage III colon carcinoma: A role for the host response in prognosis. Cancer 97: 960-968, 2003.
32. Strojnik T, Kavalar R, Zajc I, Diamandis EP, Oikonomopoulou K and Lah TT: Prognostic impact of CD68 and kallikrein 6 in human glioma. Anticancer Res 29: 3269-3279, 2009.

33. Solinas G, Germano G, Mantovani A and Allavena P: Tumorassociated macrophages (TAM) as major players of the cancer-related inflammation. J Leukoc Biol 86: 1065-1073, 2009.

34. Delgado M, Abad C, Martinez C, Leceta J and Gomariz RP Vasoactive intestinal peptide prevents experimental arthritis by downregulating both autoimmune and inflammatory components of the disease. Nat Med 7: 563-568, 2001.

35. Delgado $M$ and Ganea D: Inhibition of IFN- $\gamma$-induced janus kinase-1-STAT1 activation in macrophages by vasoactive intestinal peptide and pituitary adenylate cyclase-activating polypeptide. J Immunol 165: 3051-3057, 2000.

36. Ganea D and Delgado M: Vasoactive intestinal peptide (VIP) and pituitary adenylate cyclase-activating polypeptide (PACAP) as modulators of both innate and adaptive immunity. Crit Rev Oral Biol Med 13: 229-237, 2002.

37. van Horssen R, Ten Hagen TL and Eggermont AM: TNF- $\alpha$ in cancer treatment: Molecular insights, antitumor effects, and clinical utility. Oncologist 11: 397-408, 2006.

38. Krejs GJ: VIPoma syndrome. Am J Med 82B: 37-48, 1987. 